\title{
Redes neuronales (RN) aplicadas a la valoración de locales comerciales
}

\section{Neural networks (NN) applied to the commercial properties valuation}

\author{
J. M. Núñez Tabales $^{(*)}$, F. J. Rey Carmona ${ }^{(*)}$, J. M. $^{\text {a }}$ Caridad y Ocerin ${ }^{(*)}$
}

\section{RESUMEN}

La estimación de precios de inmuebles mediante la utilización de métodos objetivos es de interés para compradores, vendedores y para la propia Administración. Existen diferentes metodologías que permiten la determinación del precio de un inmueble, siendo numerosas las aportaciones cuyo propósito es la estimación de precios de inmuebles residenciales. No obstante, el presente trabajo es pionero en la aplicación de técnicas de Inteligencia Artificial (IA) para la determinación de precios de locales comerciales. Se presenta un estudio de esta tipología de inmueble en la ciudad de Córdoba (España). Los resultados evidencian que las Redes Neuronales ( $\mathrm{RN}$ ) constituyen una alternativa atractiva a los tradicionales Modelos Hedónicos ( $\mathrm{MH}$ ), registrando un mejor ajuste a las no linealidades del mercado y resultando con menores errores. Asimismo, se obtienen los precios implícitos correspondientes a los atributos determinantes del precio de un local comercial a partir de la ecuación de la $\mathrm{RN}$ diseñada.

Palabras clave: Inteligencia Artificial (IA); Redes Neuronales (RN); precio local comercial; modelos econométricos; valoración inmobiliaria.

\section{ABSTRACT}

Several agents, such as buyers and sellers, or local or tax authorities need to estimate the value of properties. There are different approaches to obtain the market price of a dwelling. Many papers have been produced in the academic literature for such purposes, but, these are, almost always, oriented to estimate hedonic prices of residential properties, such as houses or apartments. Here these methodologies are used in the field of estimate market price of commercial premises, using AI techniques. A case study is developed in Cordova - city in the South of Spain-. Neural Networks are an attractive alternative to the traditional hedonic modelling approaches, as they are better adapted to non-linearities of causal relationships and they also produce smaller valuation errors. It is also possible, from the NN model, to obtain implicit prices associated to the main attributes that can explain the variability of the market price of commercial properties.

Keywords: Artificial Intelligence (AI); Neural Networks (NN); commercial property price; econometric modelling; real estate valuation.

(*) Universidad de Córdoba (Córdoba, España).

Persona de contacto/Corresponding author: es2nutaj@uco.es (J. M. Núñez)

ORCID: http://orcid.org/oooo-0oo1-6597-6029 (J. M. Núñez Tabales); http://orcid.org/oooo-0002-2434-556X

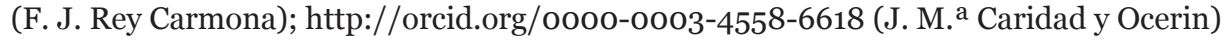

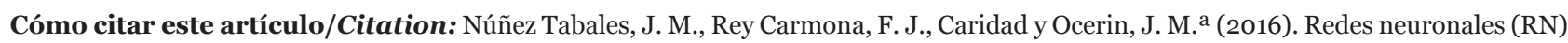
aplicadas a la valoración de locales comerciales. Informes de la Construcción, 69(545): e179, doi: http://dx.doi.org/10.3989/ic.15.053.

Copyright: (C) 2017 CSIC. Licencia / License: Salvo indicación contraria, todos los contenidos de la edición electrónica de Informes de la Construcción se distribuyen bajo una licencia de uso y distribución Creative Commons Attribution License (CC BY) Spain 3.o. 


\section{INTRODUCCIÓN: IMPORTANCIA DE LA VALORACIÓN INMOBILIARIA Y METODOLOGÍAS APLICABLES}

La valoración ha estado presente a lo largo de la historia de la humanidad. En nuestros días, en multitud de actos económicos es necesario conocer el valor de los activos implicados. La valoración inmobiliaria es una de las que más frecuentemente afecta a los ciudadanos, dado que se encuentra presente en multitud de situaciones cotidianas como, por ejemplo, el pago de tributos, la compra de un inmueble o la solicitud de un préstamo hipotecario. Desde la perspectiva de la Administración Pública, la valoración de inmuebles también es de transcendental importancia, dado que buena parte del sistema impositivo tiene su base en los distintos gravámenes que recaen sobre bienes inmuebles.

Un dato que pone de manifiesto la importancia de la valoración inmobiliaria es que en España, a lo largo del año 2013 las 52 sociedades de tasación homologadas efectuaron un total de 661.00o tasaciones, con un valor total tasado que ascendió a 265 mil millones de euros y una superficie total de los bienes inmuebles valorados en el ejercicio de 517.000 hectáreas (1).

Ahora bien, dentro del mercado inmobiliario el mercado de la vivienda es el principal componente. En consecuencia, la vivienda ha tenido en la literatura relativa a la valoración inmobiliaria un papel preponderante, mientras que el del resto de inmuebles ha sido marginal. De hecho, en el año 2013, del total de valoraciones realizadas por el conjunto de sociedades de tasación de España, las viviendas individuales - bien sean elementos de edificios o unifamiliares-supusieron el $59 \%$ del total de las realizadas (1). De esta tipología se efectuaron 389.000 tasaciones por un importe global de 69 mil millones de euros. El segundo tipo de inmueble sobre el que se efectuaron mayor número de tasaciones fue el local comercial, con 46.000 tasaciones, lo que supuso un $7 \%$ del total de las realizadas. El importe global de las tasaciones de locales comerciales fue de 12 mil millones de euros.

Por consiguiente, en el presente trabajo se ha optado por elegir al local comercial como el tipo de inmueble que constituirá el objeto de estudio por dos razones fundamentales: (i) la escasez de estudios empíricos existentes en torno a este tipo de inmueble - a pesar de su relevancia en la estructura y en la configuración de la ciudad-; (ii) el local comercial es el tipo de inmueble que de forma más frecuente es objeto de valoración, tras la vivienda por supuesto. Por lo tanto resulta de interés conocer cuáles son los principales determinantes del precio del local comercial.

En la actualidad, la valoración inmobiliaria se configura como una actividad pluridisciplinar en la que se combinan diferentes técnicas y metodologías de estudio con objeto de establecer el valor real de un producto inmobiliario.

Han sido varios los autores que han intentado efectuar una clasificación de la diversidad de métodos existentes para la valoración de un inmueble. Entre las distintas propuestas destaca la realizada por Pagourtzi et al. (2), muy considerada en la bibliografía acerca de esta temática - véase, por ejemplo, a Selim (3) y Kusan et al. (4)-. Pagourtzi clasifica los métodos en dos categorías: métodos tradicionales y métodos avanzados. Los primeros coinciden básicamente con los métodos técnicos de valoración, aunque se permite incluir entre los mismos alguna técnica matemática clásica como el análisis de regresión. Entre los métodos avanzados incluye a las Redes Neuronales Artificiales, al método de Precios Hedónicos, a los métodos de Análisis Espacial, a la Lógica Difusa y a las técnicas Box-Jenkins -Autoregressive Integrated Moving Average (ARIMA)-.

Por otra parte, hay que considerar las clasificaciones metodológicas efectuadas por Gallego (5) y Aznar (6). El primero efectúa una diferenciación entre los métodos de valoración tradicionales y los métodos de valoración automatizada. Dentro del primer grupo se incluyen aquellos basados fundamentalmente en el criterio de un experto, cuentan con un alto grado de precisión, pero se caracterizan por su elevado nivel de subjetividad y por una baja producción en cuanto al número de valoraciones. Este tipo de métodos tiene una gran aceptación por parte de particulares, empresas, entidades bancarias, organismos tributarios, etc. En un segundo grupo encuadra a los que denomina métodos de valoración automatizada $^{1}$, caracterizados fundamentalmente por el empleo de técnicas matemáticas para la estimación del valor. El empleo de este tipo de técnicas, junto con un procedimiento más sistemático, les otorga un carácter más científico, revistiéndolos de un mayor nivel de objetividad, al tiempo que facilitan una mayor producción de valores.

Aznar (6) realiza una clasificación de los métodos de valoración realizada a partir de los recogidos en las Normas Internacionales de Valoración, a los que añade los denominados métodos Multicriterio, así como distintos métodos previstos para la valoración de activos y recursos ambientales. No obstante, de entre ellos considera como los más frecuentemente utilizados en la práctica a los métodos por corrección en sus dos variantes, el método del ratio de valoración, el método Beta, el método del análisis de regresión, el método de actualización de rentas, el método del coste de reemplazamiento y el del valor residual.

En un intento de aunar las posibles clasificaciones, en la Tabla 1 se agrupan los distintos métodos de valoración de inmuebles en dos categorías, diferenciando entre métodos técnicos y métodos avanzados.

Como se ha indicado anteriormente las aportaciones en el ámbito de la valoración de locales comerciales son muy escasas. De hecho, únicamente se cuenta con una aplicación del método de los Precios Hedónicos efectuada por Humarán (7) en las principales ciudades catalanas y, por otro lado, de una aplicación del análisis espacial a la ciudad de Toledo realizado por Montero y Larraz (8) utilizando la técnica conocida como krigeage.

En el presente trabajo se desarrolla un estudio empírico de locales comerciales haciendo uso de técnicas avanzadas de valoración. Por consiguiente, este estudio tiene como objetivo principal la obtención de modelos predictivos del precio mensual de alquiler de un local comercial en la ciudad de

\footnotetext{
Esta denominación no obedece, precisa el propio autor, a la utilización de técnicas informáticas, puesto que también son empleadas en los métodos tradicionales.
} 
Tabla 1. Clasificación de los métodos de valoración inmobiliaria.

\begin{tabular}{|c|l|}
\hline \multicolumn{1}{|c|}{ Grupo } & \multicolumn{1}{c|}{ Método } \\
\hline \multirow{5}{*}{ Técnicos } & - Coste \\
& - Comparación con el mercado \\
& - Actualización de rentas \\
& Residual \\
& - Estático \\
& - Dinámico \\
\hline \multirow{5}{*}{ Avanzados } & - Inecios Hedónicos \\
& Lógica Difusa, Sistemas Expertos y Algoritmos \\
& Genéticos) \\
& - Análisis Espacial \\
& - K-Vecinos \\
& - Basados en la Teoría de decisión multicriterio \\
& - Técnicas Box-Jenkins (Modelos ARIMA) \\
\hline
\end{tabular}

Fuente: Elaboración propia.

Córdoba, ubicado en zona urbana no periférica. Se pretende así facilitar modelos de predicción de precios en el mercado de locales comerciales de la ciudad, de manera que permitan automatizar la obtención de dichos valores.

Concretamente se partirá de una serie de testigos relacionados con la modalidad de inmueble citada y a partir de ahí se construirán modelos econométricos para determinar de forma objetiva su precio de mercado. Asimismo, se pretende estimar el peso marginal de cada uno de los atributos (precios implícitos) que explican el precio del local comercial, cuantificando esa influencia de modo que se consiga valorar su importancia relativa sobre el precio.

En concreto, se utilizará la Inteligencia Artificial (IA) - Redes Neuronales (RN) - para determinar cuáles son los principales factores externos e internos que determinan el precio final de esta tipología de bien. A continuación los resultados serán comparados con los obtenidos por un método hedónico clásico.

\section{LA INTELIGENCIA ARTIFICIAL (IA) EN EL ÁMBITO DE LA VALORACIÓN INMOBILIARIA}

Siguiendo a Martín y Sanz (9) podría definirse la IA como un conjunto de algoritmos cuyo objetivo es imitar el razonamiento humano a través de una lógica deductiva o manipulación de símbolos. Por su parte, Pino et al. (10) señalan una visión parecida destacando que en el seno de la IA, desde distintas perspectivas, se han ido acumulando conocimientos sobre cómo emular las diversas capacidades del ser humano para exhibir comportamientos inteligentes y se han desarrollado sistemas cada vez más perfeccionados que reproducen parcialmente dichas capacidades. Por consiguiente, la IA es un término genérico que engloba técnicas muy diferentes unas de otras, pero que tienen en común esa imitación de procesos inteligentes existentes en la naturaleza humana.

Dentro de la IA se pueden distinguir dos grandes áreas según Isasi y Galván (11). Una es la de la IA simbólica, la que se ocupa de la construcción de sistemas que se puedan definir como «inteligentes». En este caso se define el problema a resolver y se diseña el sistema capaz de resolverlo siguiendo esquemas prefijados por la disciplina. Se dice que los sistemas siguen un esquema de arriba abajo (en inglés top - down), ya que es necesario disponer de una aproximación a la solución del problema y diseñarla completamente. La otra gran área de la IA es la subsimbólica, es el caso en que no se realizan dise- ños a alto nivel de sistemas capaces de resolver los problemas utilizando las técnicas de la disciplina, sino que se parte de sistemas genéricos que van adaptándose y construyéndose hasta formar por sí mismos un sistema capaz de resolver el problema. En este caso el diseño es de abajo hacia arriba (bottom - up), ya que los sistemas diseñados son simples e idénticos, recogen las características físicas de los sistemas que tratan de imitar y se van generando cómputos cada vez más complejos, de forma automática, mediante mecanismos prefijados de aprendizaje. Es en esta área donde se ubican las Redes Neuronales (RN). Lo que diferencia a éstas de otras metodologías es la inspiración biológica de cómo funciona el cerebro, es decir, otros métodos profundizan en los modelos del cerebro provenientes de la psicología más tradicional (qué hace el cerebro sin importar el cómo), pero las RN no se preocupan solo de conseguir un funcionamiento inteligente, sino también de las características estructurales o anatómicas de una posible máquina inteligente.

Quedarían vinculadas al ámbito de la IA técnicas como la Lógica Difusa o Borrosa (Fuzzy Logic), los Sistemas Expertos, los Algoritmos Genéticos y las ya mencionadas RN.

Los primeros estudios sobre valoración de inmuebles que utilizan la IA datan de principios de los años noventa, con los trabajos de Borst (12) en Nueva Inglaterra en 1991, por consiguiente la aplicación de estas técnicas en el ámbito de determinación de precios inmobiliarios tiene más de dos décadas de vida. La mayor parte de los sistemas de IA aplicados a este campo son RN.

Una RN está formada por un conjunto de elementos o unidades de proceso (PE) de información, interconectadas, formando un sistema para ajuste de modelos no lineales. Fueron introducidas por McCulloch y Pitts (13) en 1943. A partir de unas variables de entrada, $x_{1}, x_{2}, \ldots, x_{n}$, se trata de explicar las variaciones de la variable de salida $y-y=m\left(x_{1}, x_{2}, \ldots, x_{n}\right)+\varepsilon-$. El esquema de funcionamiento de una neurona artificial se muestra en la Ilustración 1. Los modelos de redes pueden ser multiecuacionales y utilizarse no sólo para estimación, sino también para problemas de clasificación. La dependencia de las variables de salida respecto de las de entrada se formula mediante unas variables no observables, denominadas neuronas, con una estructura o topología en una o varias capas.

Las neuronas realizan un procesamiento de la información de entrada y generan una salida a través de una función de activación o umbral. La salida es enviada a todas las neuronas de la capa siguiente, procesada mediante una función monótona definida en $[0,1]$, como la función logística o la tangente hiperbólica. La información de salida hacia la última capa permite obtener la estimación del precio, ŷ. La estimación de

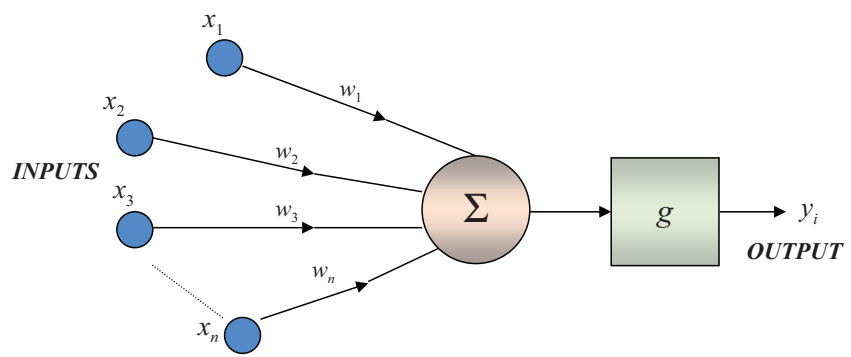

Ilustración 1. Esquema de neurona artificial o elemento de proceso (PE). Fuente: Elaboración propia. 
los distintos parámetros se realiza minimizando una función de error, como el Error Cuadrático Medio, mediante un algoritmo iterativo o proceso de aprendizaje «hacia atrás», en el que los parámetros se van modificando para obtener un valor mínimo del ECM, utilizando métodos de gradiente.

En relación a la topología de una red, es decir, a la forma en que se estructuran las distintas capas y sus conexiones, existen diversos tipos de redes. La más comúnmente utilizada en valoración de inmuebles es el Perceptrón Multicapa (Multilayer Preceptron - MLP-), formada por una capa de entrada (inputs), una de salida (output) y una (o varia(s) capa(s) oculta(s).

Determinados autores efectúan una comparativa entre técnicas tradicionales de precios hedónicos ${ }^{2}$ (especialmente análisis de regresión múltiple-MRA-) e inteligencia artificial. La gran mayoría de los trabajos apoyan la utilización de las RN por su robustez y su gran capacidad para detectar relaciones no lineales entre variables -es el caso de Tay y Ho (15); Do y Grudnitski (16); Kauko et al. (17); Peterson y Flanagan (18); Caridad et al. (19) y Cannavaro (20) - , mientras que otros ponen de manifiesto que las RN no son necesariamente superiores a los modelos de regresión -entre ellos se encuentran los de Allen and Zumwalt (21), Worzala, Lenk y Silva (22), Rossini (23), o Zurada et al. (24) - comparando su actuación en diferentes escenarios en los que llegan a distintas conclusiones acerca de la superioridad de las RN. Por su parte, Limsombunchai et al. (25) llegaron a resultados equivalentes aplicando ambas metodologías.

Por otro lado, en nuestro país las primeras aportaciones a este campo tienen algo más de una década, siendo pioneros Caridad y Ceular (26) en el año 2001 cuyo análisis se desarrolla en la ciudad de Córdoba, seguidos de otras contribuciones destacables como las de Mohamed en el año 2002 (27), Gallego en 2004 (28), García Rubio (29) en 2004, Lara (30) en 2005, o Landajo et al. (31) en 2012 en las ciudades de Cádiz, Madrid, Albacete, Jaén y Oviedo, respectivamente.

Hay que señalar que los estudios analizados (tanto a nivel nacional como internacional) versan sobre valoración de un tipo de inmueble: la vivienda (ya sea unifamiliar o plurifamiliar), no existiendo referencia alguna para la aplicación de esta metodología en la determinación de precios de locales comerciales.

Como se ha señalado con anterioridad el tipo de red más utilizada con diferencia es el Perceptrón Multicapa -Multilayer Perceptron (MLP)-, aunque algunos estudios prueban otro tipo de redes como la Función de Base Radial (RBF) -García Rubio (29) y Zurada et al. (24) - o los Mapas Auto-organizativos de Kohonen -Kauko et al. (17) y Kontrimas y Verikas (32)-.

En la capa de entrada (inputs) del MLP se sitúan las variables que se consideran determinantes del precio del inmueble. En cuanto a la capa de salida de la red (output), ésta estará constituida por un único nodo o neurona que suele ser el precio de transacción del inmueble (ya sea total o por metro cuadrado), aunque existen referencias en las que se manejan precios de oferta, así como precios de tasación o precios de alquiler.

\section{ANÁLISIS EMPÍRICO}

\subsection{Materiales utilizados}

Para ilustrar las posibilidades de las $\mathrm{RN}$ en la determinación de precios de inmuebles se ha desarrollado un caso de estudio para la ciudad de Córdoba, usando una muestra de 102 locales comerciales situados a pie de calle. Los datos de trabajo han sido facilitados por sociedades mercantiles cuyo objeto social es el alquiler de locales comerciales en la citada ciudad, usando una plantilla de recogida de datos. La información obtenida se corresponde con precios reales de alquiler mensual (Impuesto sobre el Valor Añadido no incluido), referidos todos ellos al mes de enero de 2013.

De acuerdo con los datos poblacionales manejados, la muestra seleccionada es representativa de las rentas de alquiler satisfechas en la ciudad de Córdoba en el período referenciado. Asimismo, los locales comerciales de la muestra abarcan la totalidad de las zonas comerciales definidas para dicha ciudad.

La selección de los atributos determinantes en la valoración fue una tarea ardua para la que se contó con la colaboración de diversos expertos inmobiliarios (agentes de la propiedad inmobiliaria, administradores y directores de las sociedades mercantiles de arrendamiento consultadas).

En la plantilla de recogida se manejaron un total 38 variables, de las cuales únicamente fueron seleccionadas finalmente 17 (Tabla 2). Aunque presumiblemente se pensó en un primer estadio que las variables iniciales podrían influir en el precio del inmueble, bien se ha observado un resultado homogéneo o bien no se ha podido precisar su valor en un número importante de casos, por lo que se descartan como variables determinantes en un análisis ulterior. Es preciso indicar que entre las variables recogidas en la Tabla 2 se encuentran todas aquellas consideradas como de especial relevancia por los expertos inmobiliarios consultados.

La mayoría de las variables manejadas son de carácter cuantitativo. No obstante, han sido categorizadas para poder ser tratadas estadísticamente: la calidad de los pavimentos y la fachada del inmueble, la zona de ubicación (que indica el barrio o área comercial al que pertenece el local), la calidad de ubicación dentro de la zona (que mide el atractivo comercial de su ubicación exacta), la posición en esquina, la forma geométrica o el uso del local.

Con el objetivo último de complementar la información obtenida mediante la plantilla inicial, se incorpora información acerca de aspectos concretos que pueden influir en la valoración del inmueble y que pueden inferirse a partir de la ubicación del mismo. Utilizando la información proporcionada por el Excmo. Ayuntamiento de Córdoba, la Cámara de Comercio y el callejero Fiscal, concretamente se consideró de interés incluir en la base de datos las siguientes variables: Zona vecinal, Renta de la zona, Código Postal, Número de actividades en cada Código Postal, Número de actividades en cada zona, Población en cada zona y Densidad de población por actividad en cada zona.

\footnotetext{
2 El método de los Precios Hedónicos parte de la premisa de que si un bien está en realidad constituido por un conjunto de atributos, entonces su precio de mercado deberá ser un agregado de los precios individuales de todos ellos. Su origen puede situarse en Haas (14) en 1922, quien efectuó un análisis de los precios de la tierra de cultivo.
} 
Tabla 2. Clasificación de atributos del local comercial.

\begin{tabular}{|l|l|l|l|}
\hline \multicolumn{2}{|c|}{ Internos } & \multicolumn{1}{|c|}{ Externos } \\
\hline Básicos & $\begin{array}{l}\text { Superficie } \\
\text { Metros lineales totales (principales, secundarios y fondo) } \\
\text { Metros lineales traslúcidos (principales y secundarios) } \\
\text { Forma geométrica }\end{array}$ & Generales & Año edificación \\
\hline Generales & $\begin{array}{l}\text { Calidad pavimentos } \\
\text { Calidad fachada }\end{array}$ & $\begin{array}{l}\text { Localización } \\
\text { Erecio de mercado } \\
\text { Ectividad económica (uso del local) }\end{array}$ & $\begin{array}{l}\text { Zona ubicación } \\
\text { Calidad dentro de la zona } \\
\text { Esquina }\end{array}$ \\
\hline
\end{tabular}

Para trabajar con variables de carácter cualitativo se construyeron índices que incluyen simultáneamente varios atributos. Estos índices toman valores que oscilan entre o y 1 (intervalo cerrado) con objeto de homogeneizarlos. De manera que se pone de manifiesto una situación más favorable a medida que el índice se aproxima a la unidad. Los tres índices construidos se recogen en la Tabla 3 .

\subsection{Especificación del modelo}

Como paquete estadístico para la determinación de la RN se eligió el programa Trajan Neural Networks 6.o. Tras efectuar numerosas pruebas con distintos tipos de red, finalmente se seleccionó un Perceptrón Multicapa -MLP (Multi Layer Preceptron)-.

En el diseño de la arquitectura de la red neuronal se barajaron diversas combinaciones en cuanto a: número de capas ocultas, número de neuronas por capa, funciones de activación y algoritmo de entrenamiento. Los detalles de la RN seleccionada pueden visualizarse de forma resumida en la Tabla 4.

Los inputs que se usarán en la capa de entrada de la red son las variables recogidas en el trabajo de campo correspondientes a la construcción de la base de datos de locales comerciales descrita

Tabla 3. Indicadores sintéticos aritméticos.

\begin{tabular}{|l|l|}
\hline \multicolumn{1}{|c|}{ Índice } & \multicolumn{1}{c|}{ Características incluidas } \\
\hline $\begin{array}{l}\text { Índice de } \\
\text { CONSERVACIÓN }\end{array}$ & Pavimento y fachada \\
\hline $\begin{array}{l}\text { Índice de } \\
\text { VISUALIZACIÓN }\end{array}$ & $\begin{array}{l}\text { Calidad de ubicación dentro de la zona y } \\
\text { posición en esquina }\end{array}$ \\
\hline $\begin{array}{l}\text { Índice de } \\
\text { UBICACIÓN }\end{array}$ & $\begin{array}{l}\text { Barrio donde se ubica el edificio y nivel de } \\
\text { renta de la zona }\end{array}$ \\
\hline
\end{tabular}

Tabla 4. Características de la RN seleccionada.

\begin{tabular}{|l|c|}
\hline Arquitectura & \multicolumn{1}{|c|}{$5: 5-6-1: 1$} \\
\hline Neuronas en capa entrada & 5 \\
\hline Neuronas ocultas & 6 \\
\hline Neuronas en capa salida & 1 \\
\hline Número de pesos & $43(36+7)$ \\
\hline Función de activación & Lineal-Logística-Logística \\
\hline Función de error & $\begin{array}{l}\text { Suma de cuadrados de los } \\
\text { errores }\end{array}$ \\
\hline Algoritmo de entrenamiento & $\begin{array}{l}\text { Retropropagación de } \\
\text { errores (BP) y Gradientes } \\
\text { conjugados(CG) }\end{array}$ \\
\hline
\end{tabular}

anteriormente. No obstante, aunque se probó con multitud de combinaciones de variables, los inputs finalmente seleccionados fueron: la superficie del local, índice de conservación, índice de ubicación, antigüedad del edificio en que se ubica, así como la interacción entre la calidad de la ubicación y la posición en esquina.

La arquitectura elegida $(5: 5-6-1: 1)^{3}$ contiene cinco inputs o neuronas en la capa de entrada, seis en la capa oculta y una única neurona en la capa de salida, pues se pretende efectuar la tarea de regresión con una única variable dependiente (el precio del local). El número de neuronas en la capa oculta se fijó tras efectuar multitud de pruebas hasta finalmente dejar seis neuronas en dicha capa, lo que origina un total de 43 pesos ( 36 hasta la capa oculta y otros 7 hasta la capa de salida), de los cuales 7 son umbrales.

La muestra fue dividida en dos subconjuntos de forma aleatoria, con objeto de garantizar la capacidad generalizadora de la red $-80 \%$ para la fase de entrenamiento y el resto se reservó para el test-.

Para efectuar el entrenamiento de la red se combinaron dos algoritmos: en una primera fase se usó el algoritmo de retroprogagación de errores -BP o Backpropagation-y en la segunda fase se seleccionó el de Gradientes Conjugados - CG(con máximo número de iteraciones en 200 para ambos). Además, se fijó un ratio de aprendizaje de o,01 y el término de momento en 0,3 .

El Gráfico 1 muestra la evolución del error a lo largo del proceso de entrenamiento. Un aspecto fundamental a tener pre-

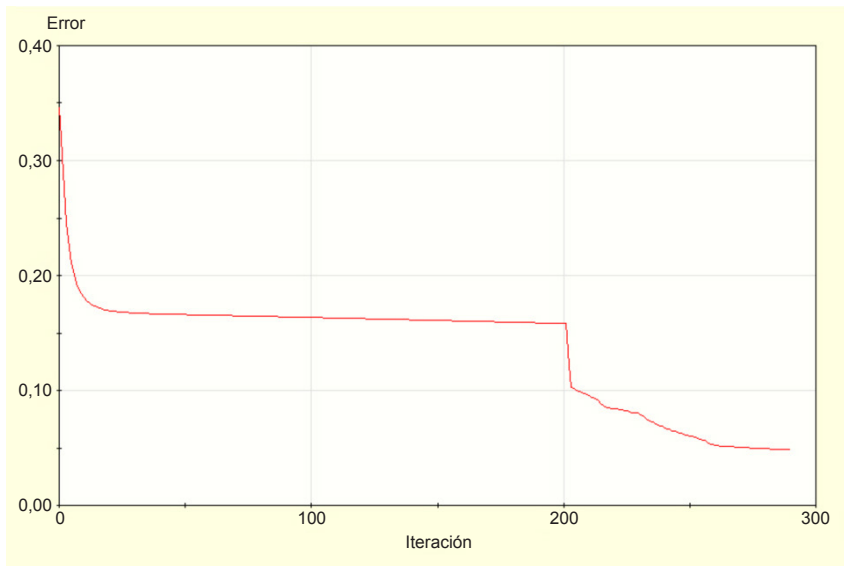

Gráfico 1. Evolución del error durante el proceso de entrenamiento.

\footnotetext{
${ }^{3}$ 5:5 significa que hay un total de cinco inputs o neuronas en la capa de entrada y que tras el preprocesamiento de los datos continúa habiendo el mismo número de neuronas en dicha capa, dado que no se han utilizado codificaciones especiales (como el esquema 1-de-N) que impliquen aumentar el número de neuronas para determinadas variables de entrada de carácter cualitativo.
} 
Tabla 5. Matriz de pesos de neuronas entre capa de entrada y capa oculta.

\begin{tabular}{|l|c|c|c|c|c|c|}
\hline & $\mathbf{2 . 1}$ & $\mathbf{2 . 2}$ & $\mathbf{2 . 3}$ & $\mathbf{2 . 4}$ & $\mathbf{2 . 5}$ & \multicolumn{1}{c|}{$\mathbf{2 . 6}$} \\
\hline Umbral & 1,756416 & 12,16768 & 2,53863 & 1,068854 & $-1,806674$ & 1,644023 \\
\hline Superficie & 0,2196185 & 4,626281 & 0,8645536 & $-2,424797$ & $-11,84107$ & 0,6873269 \\
\hline Iubicación & $-0,2759332$ & 2,540661 & $-0,831218$ & $-0,1748538$ & $-1,342737$ & 0,9653364 \\
\hline Antigüedad & $-1,368104$ & 3,047515 & $-1,313245$ & $-0,3981089$ & $-1,256938$ & 2,731032 \\
\hline Calubic $^{\wedge}$ esquina & $-1,594774$ & 4,066356 & $-1,237374$ & $-1,948007$ & $-0,4956912$ & 0,906905 \\
\hline Iconservación & $-1,974467$ & 2,709747 & $-1,088052$ & 3,284313 & $-2,446896$ & 4,350388 \\
\hline
\end{tabular}

Tabla 6. Matriz de pesos de neuronas entre capa oculta y capa de salida.

\begin{tabular}{|c|c|}
\hline & $\mathbf{3 . 1}$ \\
\hline Umbral & $-0,5769387$ \\
\hline $\mathbf{2 . 1}$ & 0,8048407 \\
\hline $\mathbf{2 . 2}$ & 11,66988 \\
\hline $\mathbf{2 . 3}$ & 0,5521522 \\
\hline $\mathbf{2 . 4}$ & $-0,8528032$ \\
\hline $\mathbf{2 . 5}$ & $-6,908442$ \\
\hline $\mathbf{2 . 6}$ & $-1,497056$ \\
\hline
\end{tabular}

sente es que la red no incurra en sobreaprendizaje que obstaculice su capacidad generalizadora. Por lo tanto, el proceso de aprendizaje finaliza en la segunda fase de entrenamiento (Gradientes Conjugados) en la iteración 91, punto en que se alcanza el mínimo error de validación.

Las matrices de pesos y umbrales correspondientes a las neuronas entre la capa de entrada y la capa oculta se observan en la Tabla 5 -la notación 2 hace referencia a la capa oculta y aparece seguido de otro número (separado por un punto) que corresponde al número de neurona de dicha capa-. Por su parte, los pesos correspondientes a las neuronas que conectan la capa oculta con la capa de salida se reflejan en la Tabla 6 -la notación para la neurona de salida es 3.1-.

Seguidamente se procede al cálculo de la ecuación de la red estimada, dado que el modelo ya ha sido validado y se ha analizado su comportamiento general. La ecuación permitirá obtener el valor de renta de cualquier local, es decir, servirá para ejecutar casos nuevos.

Como se indicó con anterioridad, el tipo de red elegida es un Perceptrón Multicapa (MLP), alimentada hacia adelante y con una única capa oculta. Su funcionamiento puede estructurarse en dos etapas:

1. ${ }^{\mathrm{a}}$ La información se propaga desde la capa de entrada a la capa oculta, calculándose la salida de las neuronas ocultas.

2. ${ }^{\mathrm{a}}$ La información se propaga desde la capa oculta hasta la capa de salida, calculándose la salida global de la red.

La notación utilizada para desarrollar la ecuación de red es la siguiente:

$-x_{i,} i=0, \ldots, n ; z_{j}, j=0, \ldots, m ; y_{k}, k=1, \ldots, l$, representan a las neuronas de la capa de entrada, oculta y de salida, respectivamente.

- $\omega_{i j}$ constituye la ponderación de la neurona $i$ de entrada en la neurona o nodo $j$ de la capa oculta $\mathrm{y} \omega^{\prime}{ }_{j k}$, la ponderación del nodo oculto $j$ en el nodo de salida $k$.

A continuación se desarrolla la operativa de las dos etapas señaladas anteriormente:
1. ${ }^{\text {a }}$ En una primera etapa se calculan las salidas de las neuronas de la capa oculta como respuesta a un vector de entradas. Usando la siguiente fórmula se obtiene la entrada neta que recibe el elemento j de la capa oculta:

$$
a_{j}=\sum_{i=0}^{n} \omega_{i j} x_{i}
$$

donde se ha considerado un peso adicional $\left(\omega_{0 j}\right)$ que actúa como umbral y pondera a una entrada fija, $x_{0}$, de valor unitario.

$$
z_{j}=f\left(a_{j}\right)
$$

La respuesta de la neurona oculta se obtiene introduciendo la entrada neta calculada dentro de la función de activación:

Para este caso concreto las funciones de activación utilizadas han sido de tipo logístico.

2. ${ }^{\text {a }}$ En la segunda etapa se obtiene la salida global de la red. De tal manera que el elemento $k$ de la capa de salida, que recibe como entradas las salidas proporcionadas por $m$ neuronas ocultas le correspondería una entrada neta de:

$$
a_{k}^{\prime}=\sum_{j=0}^{m} \omega_{j k}^{\prime} z_{j} ; z_{o}=0
$$

Por consiguiente, la salida global de la red sería:

$$
y_{k}=g\left(a_{k}^{\prime}\right)=g\left(\sum_{j=0}^{m} \omega_{j k}^{\prime} z_{j}\right)=g\left[\left[\sum_{j=0}^{m} \omega_{j k}^{\prime} f\left(\sum_{i=0}^{n} \omega_{i j} x_{i}\right)\right] \mathrm{r}\right.
$$

A partir de la función de red expresada, las matrices de pesos y umbrales recogidas en las Tablas 5 y 6 , así como los factores de cambio de origen y escala (Tabla 7.48) para la normalización de las variables manejadas, es posible obtener el precio mensual del alquiler de cualquier local comercial.

La Tabla 7 recoge los factores de cambio de origen y escala tanto de las variables de entrada como de la variable de salida.

\subsection{Resultados}

Para observar la influencia que posee cada uno de los inputs seleccionados sobre el output se efectúa el análisis de sensi-

Tabla 7. Factores de preprocesamiento y postprocesamiento de los inputs y output.

\begin{tabular}{|l|c|c|}
\hline & $\begin{array}{c}\text { Factor de cambio } \\
\text { de origen }\end{array}$ & $\begin{array}{c}\text { Factor de cambio } \\
\text { de escala }\end{array}$ \\
\hline Superficie & $-0,0134048$ & 0,00108717 \\
\hline Iubicación & 0.000000000 & 1.000000000 \\
\hline Antigüedad & $-0,04347826$ & 0,01449275 \\
\hline Calubic^esquina & $-0,25$ & 0,125 \\
\hline Iconservación & 0.000000000 & 1.000000000 \\
\hline Precio & $-0,00784658$ & 0,00012306 \\
\hline
\end{tabular}


bilidad, según el cual el ratio de error correspondiente a cada input representa el cociente entre el error del modelo sin incluir la variable y el error al incluirla. En la Tabla 8 se muestra el ratio obtenido para cada input.

Los resultados revelan que la superficie $(2,267)$ es el input que posee un mayor poder explicativo, con gran diferencia en relación al resto de inputs. A continuación se observan ratios muy parejos para las variables índice de conservación e índice de ubicación (con ratios de 1,557 y 1,537, respectivamente). Por último, los ratios más reducidos, pero con valores considerables, se obtienen para la interacción entre la calidad de la ubicación dentro de la zona y la posición en esquina $(1,494)$, así como para la antigüedad $(1,323)$. Todos los ratios obtenidos distan de la unidad, lo que denota que si elimináramos cualquiera de ellos conseguiríamos un empeoramiento de los resultados del modelo. Por lo tanto, a la vista de estas cifras no es conveniente podar la red.

En el Gráfico 2 se representa la nube de puntos correspondiente a los precios observados (eje horizontal) y los precios estimados (eje vertical). Se aprecia claramente la elevada proximidad de la mayor parte de los puntos a la bisectriz del primer cuadrante, con la excepción de tres registros que quedan infravalorados de forma notoria.

Asimismo, se efectuó una comparación de los resultados obtenidos mediante la $\mathrm{RN}$ con los de un modelo clásico Hedónico $(\mathrm{MH})$ calculado mediante un Análisis de Regresión Múltiple. Tras analizar la Tabla 9 puede afirmarse, sin lugar a dudas, que la red diseñada es capaz de aproximarse mejor a la formación del precio de un local que el modelo hedónico construido. La red neuronal posee un grado de ajuste muy superior al del modelo clásico de regresión $\left(\mathrm{R}^{2}\right.$ de $87,38 \%$ frente al 71,60\%), mayor correlación entre observaciones y estimaciones (de 0,8396 a 0,9352), así como errores cometidos muy inferiores.

Tabla 8. Análisis de sensibilidad de los inputs de la RN.

\begin{tabular}{|l|c|c|}
\hline \multicolumn{1}{|c|}{ Input } & Ratio & Orden \\
\hline Superficie & 2,267709 & 1 \\
\hline índice de conservación & 1,557319 & 2 \\
\hline índice de ubicación & 1,537454 & 3 \\
\hline Calidad de ubicación^esquina & 1,494639 & 4 \\
\hline Antigüedad & 1,323637 & 5 \\
\hline
\end{tabular}

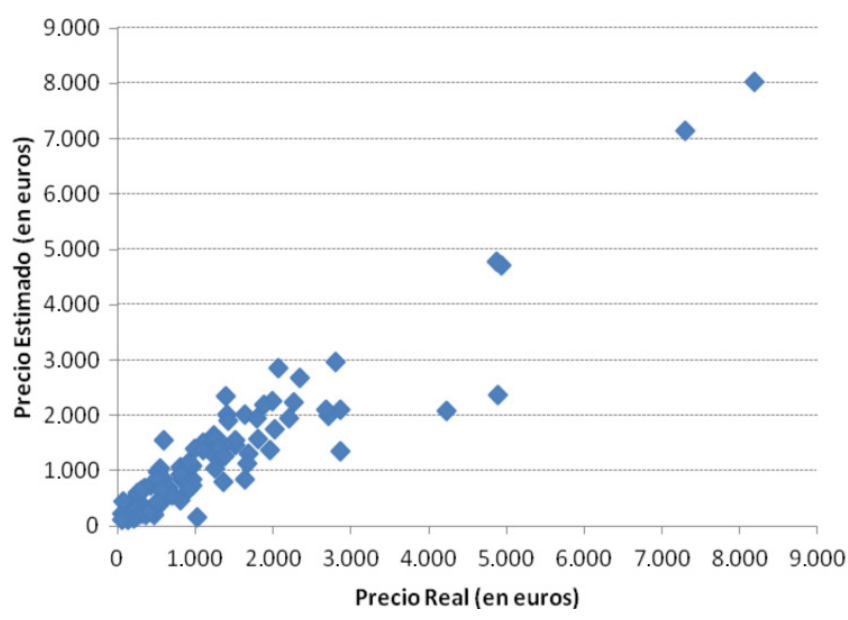

Gráfico 2. Precio real vs Precio estimado por la RN (en euros).
Tabla 9. Comparación de los resultados obtenidos con MH y RN.

\begin{tabular}{|l|c|c|}
\hline & MH & RN \\
\hline Coeficiente de Determinación (R $\left.\mathbf{( R}^{\mathbf{2}}\right)$ & $71,60 \%$ & $87,38 \%$ \\
\hline Correlación (estimada y real) & 0,8396 & 0,9352 \\
\hline $\begin{array}{l}\text { Raíz del Error Cuadrático Medio } \\
\text { (RECM) }\end{array}$ & 750,64 & 490,64 \\
\hline
\end{tabular}

Para finalizar se procedió al cálculo de los precios implícitos correspondientes a cada uno de los inputs utilizados tras la obtención de la ecuación implícita propia de la RN. Así pues, se compararon dichos precios implícitos con los provenientes del MH. Para efectuar dichos cálculos se determina la evolución del precio de los locales ante variaciones de cada uno de los inputs por separado, manteniendo todos los demás inputs constantes en sus valores medios.

En el Gráfico 3 se aprecia como el incremento marginal que se produce en el precio estimado del local a medida que aumenta la superficie del mismo es lineal creciente según el MH, es decir, ante incrementos en la superficie construida de $50 \mathrm{~m}^{2} \mathrm{se}$ producen incrementos en el precio estimado constantes e iguales a 319,25 €. Esto no tiene sentido desde un punto de vista económico, ya que el incremento en el precio no es directamente proporcional al incremento en la superficie en la realidad.

Por su parte, la RN refleja que el precio marginal que el arrendatario está dispuesto a desembolsar por el incremento en la superficie adopta forma de curva con tres partes diferenciadas: una zona cóncava (hasta los $150 \mathrm{~m}^{2}$ aproximadamente), una zona convexa (hasta alcanzar los $500 \mathrm{~m}^{2}$ aproximadamente como punto de inflexión) y, finalmente, de nuevo una zona cóncava para superficies muy grandes. Este resultado permite concluir que el arrendatario está dispuesto a pagar más por un incremento en la superficie del local hasta un determinado tamaño del inmueble (curva de rendimientos crecientes), a partir del cual el aumento de la superficie produce un aumento en los precios menos que proporcional (curva de rendimientos decrecientes) para posteriormente dar paso a incrementos en el precio cada vez mayores conforme se incrementa la superficie (curva de rendimientos crecientes para valores de más de $500 \mathrm{~m}^{2}$ ).

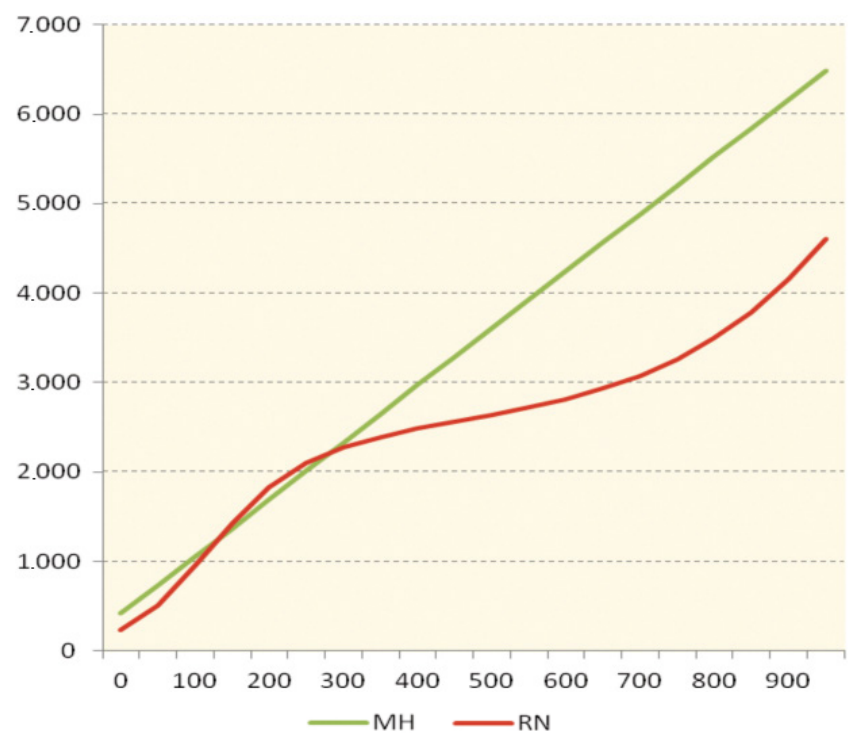

Gráfico 3. Efecto de la superficie ( $\mathrm{m}^{2}$ construido) sobre el precio estimado: $\mathrm{MH}$ vs $\mathrm{RN}$ 


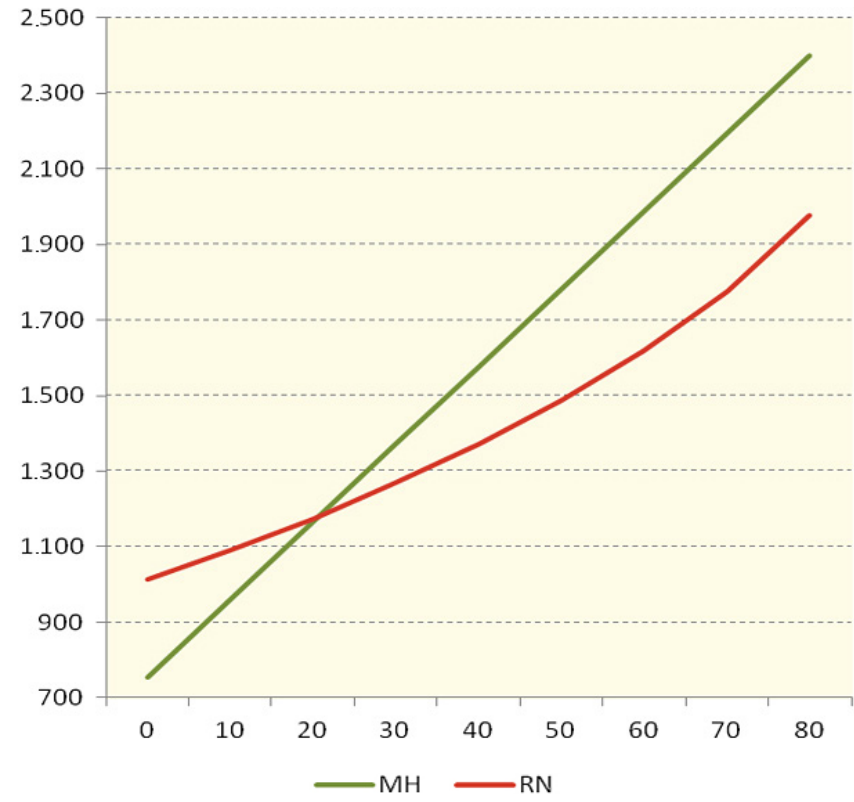

Gráfico 4. Efecto de la antigüedad sobre el precio estimado: $\mathrm{MH}$ vs RN.

En el Gráfico 4 se compara la respuesta de la $\mathrm{RN}$ y el $\mathrm{MH}$ ante variaciones de la variable antigüedad. De nuevo el modelo hedónico presenta forma lineal creciente, de forma que cada incremento de la antigüedad del inmueble en 10 años da lugar a un aumento en su precio de 205,53 €. Por su parte, la red neuronal presenta una curva ascendente de rendimientos crecientes - ante iguales incrementos en antigüedad los incrementos en el precio son cada vez mayores-.

Puede apreciarse que los precios estimados por la $\mathrm{RN}$ son mayores que los del $\mathrm{MH}$ hasta los 20 años de antigüedad aproximadamente, pero a partir de esa cifra se invierten los términos, ya que la red modera la cifra de precios.

En el Gráfico 5 se representa el efecto que posee el índice de ubicación sobre el precio estimado del local. Tal y como se señaló con anterioridad, el índice de ubicación se obtuvo pon-

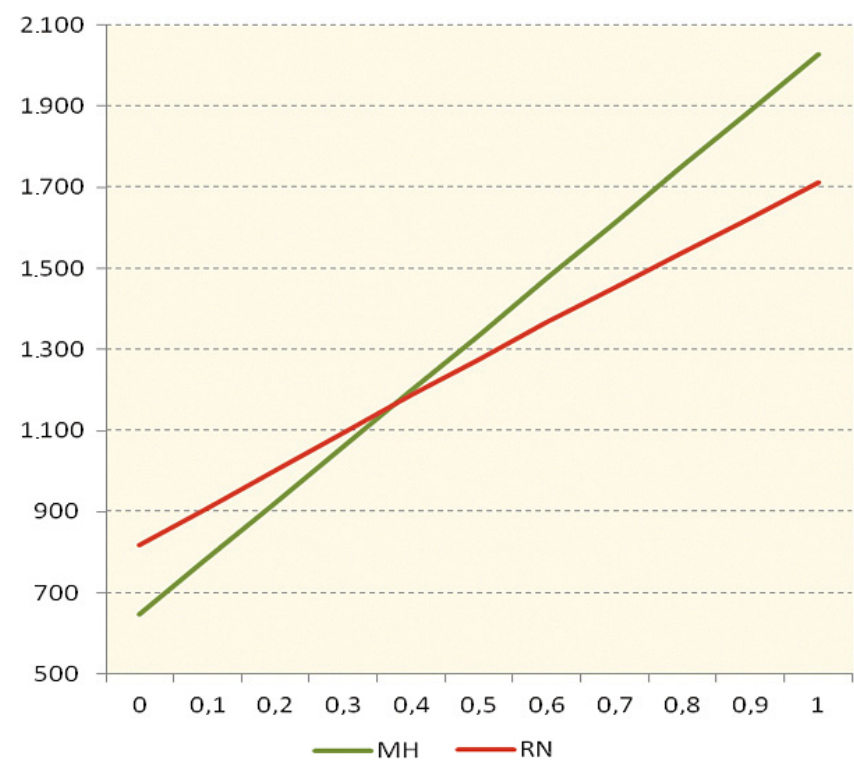

Gráfico 5. Efecto del índice de ubicación sobre el precio estimado: $\mathrm{MH} v$ s RN. derando la situación geográfica del barrio donde el local estaba ubicado y el nivel de renta de esa zona concreta, tomando valores comprendidos entre o y 1 y en aumento conforme se mejora la situación geográfica y/o el nivel de renta de la zona. En lo que respecta al precio implícito del índice de ubicación hay que destacar que el MH revela que el arrendatario está dispuesto a pagar $137,87 €$ más por cada incremento de 0,1 en dicho índice.

Según la RN el efecto del índice de ubicación sobre el precio adopta una forma casi lineal, que indica que el incremento en el precio del inmueble es prácticamente igual conforme aumenta el valor del índice de ubicación en 0,1 (los incrementos se mueven en una horquilla comprendida entre los 92 y los 86 $€)$. También se observa como los precios determinados por la red son mayores que los del modelo hedónico solamente hasta un índice de ubicación de 0,3 a partir del cual la situación es la opuesta. Por lo tanto, una vez más, la oscilación de precios que refleja el modelo hedónico por efecto de la variable índice de ubicación es mayor que la que corresponde a la red. En definitiva, la mejora del índice de o a 1 hace que el precio se incremente en $1.378 €$ según el MH, pero sólo en $893 €$ según la RN.

La representación del precio implícito para la interacción entre la calidad de la ubicación dentro de la zona y la posición en esquina (micro-localización) queda recogida en el Gráfico 6. Así pues, lo que se refleja en este caso es el producto de cinco niveles de calidad de la ubicación dentro de una zona determinada, con dos niveles que reflejan si el inmueble está o no situado en esquina. La estimación de dicho precio implícito para el $\mathrm{MH}$ es una vez más lineal (incremento de una unidad en la interacción dan lugar a aumentos constantes del precio en $137,52 €$ ). Para la $\mathrm{RN}$ se obtiene una curva ligeramente cóncava, lo que indica que el aumento en la interacción produce un aumento en los precios más que proporcional (incrementos constantes de una unidad en la interacción dan lugar a incrementos que van en aumento y oscilan entre los 62 y los $133 €)$.

Asimismo, la red nuevamente ofrece mayores precios estimados para valores reducidos en la interacción, hasta un nivel

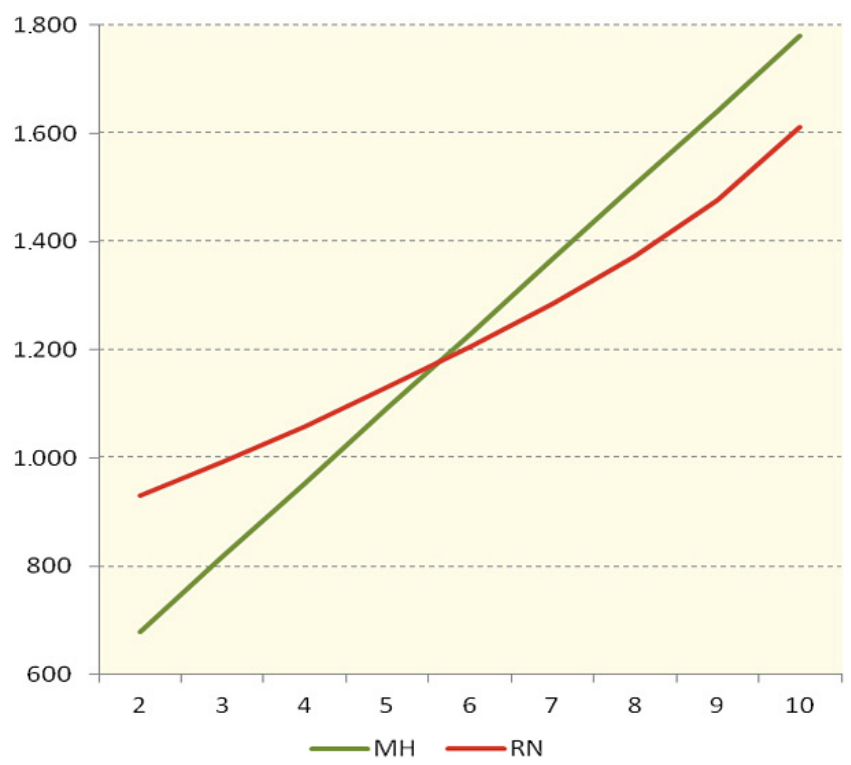

Gráfico 6. Efecto de la interacción calidad de la ubicación y posición en esquina sobre el precio estimado: $\mathrm{MH} v s \mathrm{RN}$. 


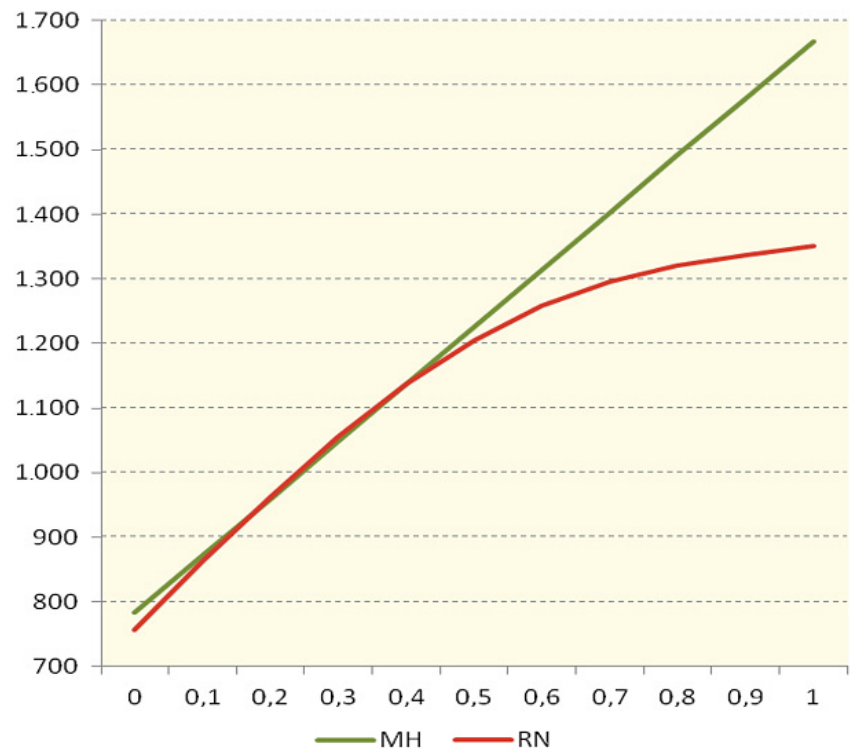

Gráfico 7. Efecto del índice de conservación sobre el precio estimado: $\mathrm{MH}$ vs RN.

de interacción de cinco, a partir de dicha cifra las estimaciones del hedónico superan a las de la red. Por lo tanto, nuevamente la red modera el efecto de esta variable sobre el precio, ya que la oscilación total que se produce en la red al estimar el precio por efecto de esta variable es de $681 €$ frente a los $1.100 €$ ofrecidos por el modelo hedónico.

En cuanto al precio implícito correspondiente al índice de conservación (construido a partir del estado y calidades de la fachada y la solería del inmueble) destacar la forma lineal del $\mathrm{MH}$ que refleja incrementos constantes de 88,44 $€$ en el precio estimado ante incrementos de 0,1 en dicho índice (Gráfico 7).

Por su parte, la RN muestra una curva convexa lo que señala que el aumento en el índice de conservación produce un aumento en los precios menos que proporcional (curva de rendimientos decrecientes con descensos comprendidos entre los $107 €$ iniciales y los $13 €$, a medida que nos acercamos al valor 1). En definitiva, hasta el índice de 0,5 el modelo hedónico y la red ofrecen precios muy parejos, como se observa claramente en el gráfico, y a partir de dicha cifra el modelo hedónico sigue su escalada proporcional, mientras que la red asciende ligeramente hasta casi estancarse en los niveles más altos del índice.

El nivel 1 en el índice de conservación queda valorado por el $\mathrm{MH}$ en 884,4 $€$, precio que puede resultar excesivo en principio y que queda suavizado por la estimación que efectúa la red en 594,3 $€$. Hay que tener presente que con la mejora del estado y las calidades de la fachada y la solería pueden ir incluidas mejoras generales en otros aspectos del inmueble como carpintería interior y exterior, pintura, techos, etc.

\section{CONCLUSIONES}

En este caso de estudio las Redes Neuronales han sido aplicadas para obtener evidencia de los factores -tanto externos como internos- que determinan el precio de un tipo de inmueble específico: el local comercial.

De los resultados alcanzados por la red propuesta para la estimación del precio local comercial en la ciudad de Córdoba, se puede concluir que las variables que mostraron poseer una mayor incidencia en el precio de los mismos fueron: la superficie, el estado conservación del inmueble -cuantificado mediante un índice que recoge el estado y calidades de la fachada y el pavimento del inmueble-, la Macro-localización en la ciudad -definida a través de un índice que pondera la situación geográfica del inmueble (barrio de la ciudad) junto con el nivel de renta de la zona-, la Micro-localización determinada por la interacción entre la calidad de ubicación del inmueble dentro de la zona concreta en que se sitúa y la posición en esquina-y, por último, la antigüedad.

Por consiguiente, la característica que más influye en la determinación del precio de alquiler del local comercial es un factor estructural como la superficie.

Sin embargo, la presencia de la variable antigüedad, así como su relación directa con la variable a explicar, fue en principio inesperada, puesto que los expertos mencionaban que en el caso de un inmueble en la modalidad de local comercial dicha variable no era considerada determinante. No obstante, en los modelos alternativos en los que se excluía este atributo se observó un descenso notable del grado de ajuste. Su presencia podría quedar justificada con los siguientes argumentos: (i) gran parte de los edificios antiguos de la ciudad están ubicados en zonas de cierta tradición o solera comercial en los que evidentemente el precio de los locales aumenta; (ii) los locales comerciales más antiguos es de esperar que hayan experimentado serias reformas en la fachada, solería, techos o carpintería, por lo que sus calidades generales pueden ser mayores que las de un local de menor antigüedad, y (iii) en un local comercial no afecta, como en una vivienda, la antigüedad del edificio debido, entre otras razones, a que para acceder a los locales analizados, todos a pie de calle, no se aprecia el estado y conservación de las zonas comunes del inmueble, ya que se accede al local directamente.

También es preciso destacar la relevancia que para el mercado de locales poseen algunas características vinculadas a la localización del mismo. El impacto de las mismas es doble. En primer lugar, se ha observado un comportamiento muy heterogéneo de los precios en las diferentes zonas de la ciudad analizadas. En segundo lugar, la calidad de la ubicación dentro de la zona y su situación en esquina también ha demostrado tener una enorme incidencia en el precio.

La metodología de $\mathrm{RN}$ se comparó con el $\mathrm{MH}$, llegando a la conclusión de que la $\mathrm{RN}$ es capaz de aproximarse mejor a la formación del precio de un local comercial que el MH. La primera opción posee un grado de ajuste muy superior al $\mathrm{MH}$, mayor correlación entre observaciones y estimaciones, así como mejores indicadores de errores.

Se calcularon además los precios implícitos correspondientes a los distintos inputs o variables explicativas. Para el MH las variaciones producidas en el precio estimado del inmueble son siempre lineales ante variaciones de cada una de las variables explicativas, situación que no se corresponde con la realidad del mercado y que la $\mathrm{RN}$ corrige por lo general de una forma más congruente.

Por todo lo anterior, se propone la $\mathrm{RN}$ estimada para la determinación del precio de un local comercial por poseer una mayor capacidad para reconocer el proceso de formación de los precios que el $\mathrm{MH}$. 


\section{REFERENCIAS}

(1) Banco de España (2014). Boletín económico, julio-agosto 2014. Las sociedades de tasación. Actividad y resultados en 2013. Madrid.

(2) Pagourtzi, E., Vassimakopoulos, V., Hatzichristos, T., French, N. (2003). Real estate appraisal: A review of valuation methods. Journal of Property Investment and Finance, 21(4): 383-401, doi: http://dx.doi.org/10.1108/ 14635780310483656.

(3) Selim, H. (2009). Determinants of house prices in Turkey: Hedonic regression versus artificial neural network. Expert Systems with Applications, 36(2): 2843-2852, doi: http://dx.doi.org/10.1016/j.eswa.2008.01.044.

(4) Kusan, H., Aytekin, O., Özdemir, I. (2010). The use of fuzzy logic in predicting house selling Price. Expert Systems with Applications, 37(3): 1808-1813, doi: http://dx.doi.org/10.1016/j.eswa.2009.07.031.

(5) Gallego Mora-Esperanza, J. (2008). Modelos de valoración automatizada. CT/Catastro, 62, abril: 7-26.

(6) Aznar Bellver, J., Guijarro Martínez, F., López Perales, A. E., González Mora, R. (2012). Valoración inmobiliaria. Métodos y aplicaciones (España e Iberoamérica). Valencia: Editorial Universitat Politècnica de València.

(7) Humarán, I., Marmolejo, C., Ruiz, M. (2008). La Formación Espacial de los Valores Comerciales, Un Análisis para las Principales Ciudades Catalanas. Ponencia presentada en XXXIV Reunión de Estudios Regionales. Baeza (Jaén): Asociación Española de Ciencia Regional.

(8) Montero, J. M., Larraz, B. (2011). Interpolation Methods for Geographical Data: Housing and Commercial Establishment Markets. Journal of Real Estate Research, 33(2): 233-244.

(9) Martín del Brío, B., Sanz Molina, A. (2006). Redes Neuronales y Sistemas Borrosos. Madrid: Ed. Ra-Ma.

(10) Pino Díez, R., Gómez Gómez, A., De Abajo Martínez, N. (2001). Introducción a la inteligencia artificial: Sistemas expertos, redes neuronales artificiales y computación evolutiva. Oviedo: Servicio de Publicaciones de la Universidad de Oviedo.

(11) Isasi Viñuela, P., Galván León, I. M. (2004). Redes neuronales artificiales: Un enfoque práctico. Madrid: Ed. Pearson Educación.

(12) Borst, R. (1991). Artificial Neural Networks: The next modelling/calibration technology for the assessment community? Property Tax Journal, 10(1): 69-94. IAAO.

(13) McCulloch, W. S., Pitts, W. (1943), A logical calculus of the ideas immanet in nervous activity. Bulletin of Mathematical Biophysics, 5: 115-133.

(14) Haas, G. C. (1922). A statistical analysis of farm sales in blue earth county, Minnesota, As a Basis for Farmland Appraisal (Masters Thesis). University of Minnesota.

(15) Tay, D. P., Ho, D. K. (1992). Artificial intelligence and the mass appraisal of residential apartment. Journal of Property Valuation \& Investment, 10: 525-540, doi: http://dx.doi.org/10.1108/14635789210031181.

(16) Do, A., Grudnitski, G. (1992). A Neural Network Approach to residential property appraisal. The Real Estate Appraiser, 58(3): 38-45.

(17) Kauko, T., Hooimaijer, P., Hakfoort, J. (2002). Capturing housing market segmentation: An alternative approach based on neural network modeling. Housing Studies, 17(6): 875-894, doi: http://dx.doi.org/10.1080/02673030215999.

(18) Peterson, S., Flanagan, A. (2009). Neural Network Hedonic Pricing Models in Mass Real Estate Appraisal. Journal of Real Estate Research, 31(2): 147-164.

(19) Caridad, J. M., Núñez J., Ceular N. (2008). Metodología de Precios Hedónicos vs Redes Neuronales Artificiales como alternativa a la valoración de inmuebles. CT/Catastro, 62: 27-42.

(20) Canavarro Teixeira, M. C. (2011). Modelos econométricos para el precio de los inmuebles: un caso de estudio en Portugal (Tesis doctoral). Córdoba: Servicio de Publicaciones de la Universidad de Córdoba.

(21) Allen, W. C., Zumwalt, J. K. (1994). Neural Networks: a word of caution. Working Paper. Colorado State University.

(22) Worzala, E., Lenk, M., Silva, A. (1995). An exploration of neural networks and its application to real estate valuation. Journal of Real Estate Research, 10(2): 185-201.

(23) Rossini, P. (1997). Artificial Neural Networks Versus Multiple Regression in the valuation of residential property. Australian Land Economics Review, 3(1): 1-12.

(24) Zurada, J., Levitan, A. S., Guan, J. (2011). A comparison of regression and artificial intelligence methods in a mass appraisal context. Journal of Real Estate Research, 33(3): 349-387.

(25) Limsombunchai, V., Gan, C., Lee, M. (2004). House price prediction: Hedonic Price Model Vs Artificial Neural Network. American Journal of Applied Sciences, 3(1): 193-201.

(26) Caridad, J. M., Ceular, N. (2001). Un análisis del mercado de la vivienda a través de redes neuronales artificiales. Estudios de Economía Aplicada, 18: 67-81.

(27) Mohamed Amar, R. (2002). Estimación del precio de la vivienda urbana mediante redes neuronales artificiales. Estudio de un caso. En XII Jornadas Hispano-Lusas. Covilhà (Portugal).

(28) Gallego Mora-Esperanza, J. (2004). La inteligencia artificial aplicada a la valoración de inmuebles. Un ejemplo para valorar Madrid. CT/Catastro, 50, abril: 51-67.

(29) García Rubio, N. (2004): Desarrollo y aplicación de redes neuronales artificiales al mercado inmobiliario: aplicación a la ciudad de Albacete (Tesis doctoral). Universidad de Castilla-La Mancha.

(30) Lara Cabeza, J. (2005). Aplicación de las redes neuronales artificiales al campo de la valoración inmobiliaria. Mapping, 104: 64-71.

(31) Landajo, M., Bilbao, C., Bilbao, A. (2012). Non parametric neural networks modeling of hedonic prices in the housing market. Empirical Economics, 42(3): 989-1009, doi: http://dx.doi.org/10.1007/s00181-011-0485-9.

(32) Kontrimas, V., Verikas, A. (2011). The mass appraisal of the real estate by computational intelligence. Applied Soft Computing, 11(1): 443-448, doi: http://dx.doi.org/10.1016/j.asoc.2009.12.003. 